This chapter has been revised in response to editorial feedback.

APA Citation: Ledgerwood, A., da Silva Frost, A., Kadirvel, S., Maitner, A. T., Wang, Y. A., \& Maddox, K. B. (in press). Methods for advancing an open, replicable, and inclusive science of social cognition. Chapter to appear in K. Hugenberg, K. Johnson, \& D. E. Carlston (Eds), Oxford Handbook of Social Cognition.

Methods for Advancing an Open, Replicable, and Inclusive Science of Social Cognition

Alison Ledgerwood ${ }^{1}$

Aline da Silva Frost ${ }^{1}$

Sanjana Kadirvel ${ }^{2}$

Angela T. Maitner ${ }^{3}$

Y. Andre Wang ${ }^{4}$

Keith B. Maddox ${ }^{2}$

${ }^{1}$ UC Davis

2 Tufts University

${ }^{3}$ American University of Sharjah

${ }^{4}$ University of Toronto 


\section{Methods for Advancing an Open, Replicable, and Inclusive Science of Social Cognition}

Imagine, for a moment, that we're all standing around a dark room. Our job is to describe an unknown sculpture at the center of it. What enables us to describe that sculpture well? We'll want good-quality flashlights and careful note-taking. We'll want people to shine their light around in various directions, rather than describing the same small spot over and over again. And we'll want people examining the statue from multiple vantage points, so that we can learn not just what the sculpture looks like on this side of the room, but what it looks like from all sides.

In this chapter, we argue that social cognition is like that sculpture. If we are to understand it, we need to employ methods and practices that allow us to perceive it clearly, to know about more than one small spot, and to approach it from multiple vantage points. We also need to get better at knowing what we do not know (Griffin \& Tversky, 1992; Sanchez \& Dunning, 2018), so that we can distinguish between solid and tentative findings and focus effort and resources on filling in the many aspects of that metaphorical sculpture that are missing from the literature. Moreover, on principle, anyone who wants to pick up a flashlight and investigate the sculpture should be able to do so. In what follows, we discuss some of the tools that scientists can use to contribute to a systematic and cumulative body of knowledge of how people think about other people.

This chapter is divided into four sections. In the first section, we describe concrete practices that researchers can use to calibrate their confidence in a significant finding to the actual strength of evidence provided by that finding. In the second section, we discuss how researchers can calibrate the scope of the conclusions they draw from a study to the stimuli and participants sampled in that study. In the third section, we highlight the importance of approaching scientific inquiry from diverse vantage points, and provide specific 
recommendations for enhancing diversity and inclusion in social cognition research. In the fourth section, we offer strategies that researchers in any career stage or role can use to help change our incentive structures so that they promote and support the practices recommended in the earlier sections. Together, these four sections offer researchers a roadmap for enhancing what we learn from the work that we do.

\section{Part I: Is It Signal or Noise? Calibrating Our Confidence in a Research Finding}

In order to build a cumulative understanding of social cognition, it is paramount to be able to calibrate our sense of what we do and do not know to the evidence we actually have. In Part I, we describe how to calibrate one's confidence that a given significant result reflects a true effect. As researchers, if a study finding is strong, we want to be relatively confident about it; if a finding is weak, we want to be more skeptical. Otherwise, we may waste resources trying to build on an effect that was in fact a false positive, or waste opportunities to invest in an interesting research program because of a false negative. In order to calibrate our confidence appropriately, we need to understand both the likely Type I error rate (the chance of observing a significant result when no actual effect exists in the population) and the likely statistical power of a test (the chance of observing a significant result when a true effect does exist in the population).

The reason to consider both Type I error and statistical power is because both together affect the positive predictive value of a finding, or the likelihood that a significant result reflects a true effect. In other words, the informational value of a significant result depends on both the power and the Type I error rate of a statistical test. The higher the power and the lower the Type I error rate, the more confident researchers can be that a given significant result reflects a true 
effect in the population. ${ }^{1}$ To get an intuitive understanding for why this is the case, let's imagine a researcher named Mei who is especially fond of apples. Mei is testing ten true effects, which we will imagine as good apples, and ten false effects, which we will imagine as wormy apples. She is going to sort these effects into two piles: Significant results (we will call this her dinner plate) and nonsignificant results (we will call this her trash bin).

Let's imagine that Mei starts by sorting the wormy apples, and that her Type I error rate is $30 \%$. That means that she is going to test each of these wormy apples (each of these false effects), and three out of ten are going to go onto her dinner plate. She makes a mistake $30 \%$ of the time. In Figure 1 (left side), we can see what happens: She has three wormy apples on her plate and seven in the trash.

Next, Mei sorts the delicious, good apples, and let's imagine that her power to correctly detect a good apple is only $30 \%$. That means that she is going to test each of these good apples (each of these true effects), and three out of the ten are going to go onto her dinner plate. She detects a true edible apple only $30 \%$ of the time. As Figure 1 (left side) illustrates, Mei ends up with three wormy apples and three good apples on her dinner plate.

Mei is not a fan of this arrangement: Half of her plate is wormy apples. She wants to be able to take a bite from an apple on her plate and be confident that it's good, but right now, she is just as likely to chomp down on a worm as she is to chomp down on an apple. She decides to start over and sort the effects using a different approach.

\footnotetext{
${ }^{1}$ For readers who find formulas helpful: The formula for positive predictive value (PPV) is ((Power*R) / (Power*R $+\alpha$ ), where $\mathrm{R}$ (the pre-study odds) is the odds of an observed effect indeed being non-null among the effects being tested, depending on the base rate of true effects (see Button et. al., 2013; Pashler \& Harris, 2012; Ioannidis, 2005). This formula is derived from a two-by-two table of presence and non-presence of non-null effects and significant and non-significant research findings. From the formula, we can see that PPV is affected by both Type I and Type II error rates: For a test with a given pre-study odds, the lower the power (1- Type II error) and the higher the Type I error, the lower the PPV.
} 
This time, Mei sets her power to correctly detect a good apple at $90 \%$ instead of only $30 \%$. Now, when she tests each of the ten good apples, nine of the ten go onto her dinner plate. As illustrated in Figure 1 (right side), this approach means that her plate contains three wormy apples and nine good apples. When taking a bite of something from her plate, Mei will still occasionally get a worm, but she can trust her plate far more in this scenario than she could in the previous one.

\section{Figure 1}

\section{An Intuitive Illustration of Positive Predictive Value}

Mei's approach to sorting the apples determines how much she can trust what is on her plate

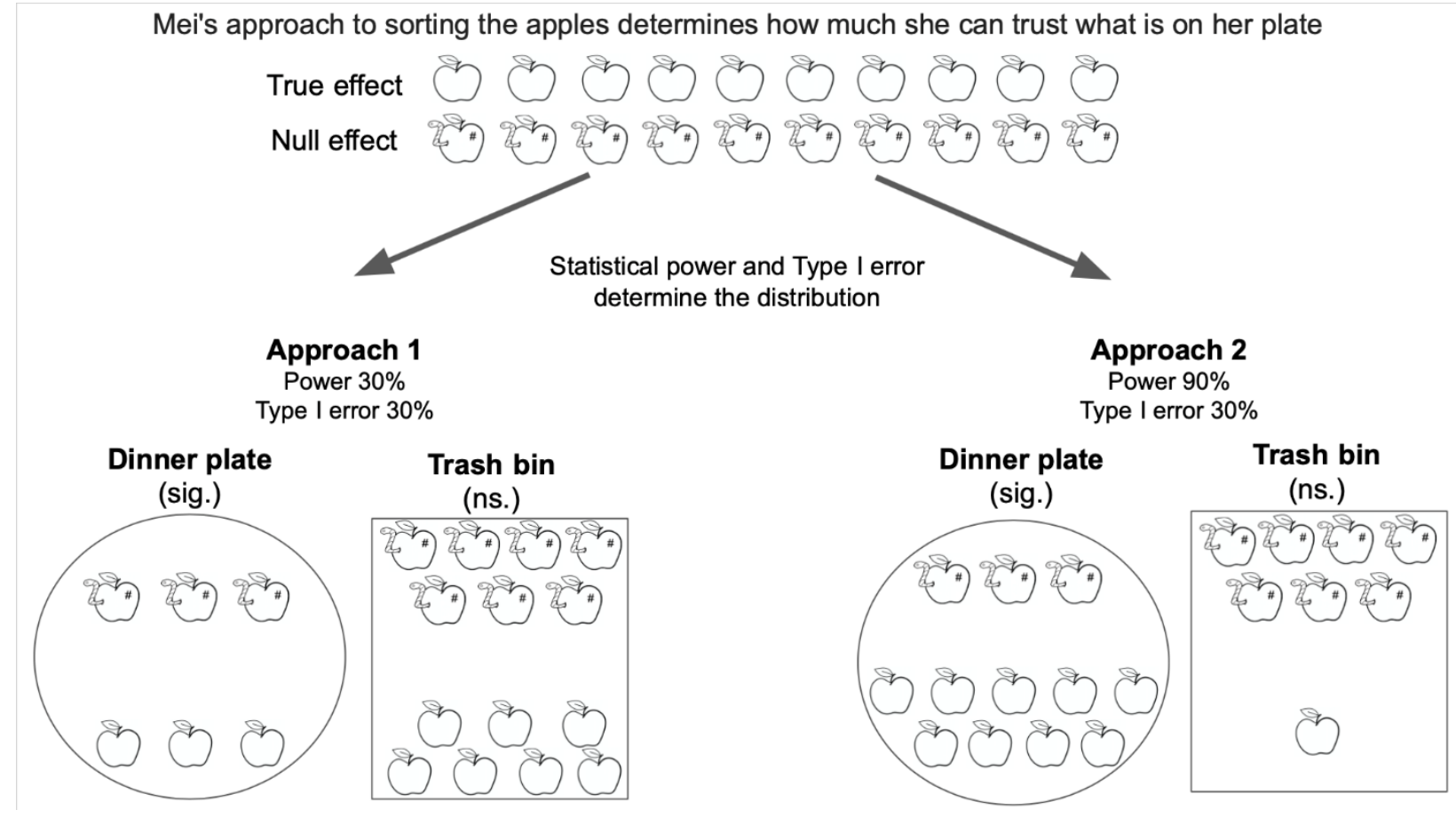

In this example, the way that Mei sorted the wormy apples was based on her Type I error rate, and the way that she sorted her good apples was based on power. Together, these two quantities influenced how much she could trust her dinner plate to contain edible food. We can think of that dinner plate as all the studies Mei will publish in her career or as the literature in a 
given topic area. The higher the power and the lower the Type I error rate, the more confident we can be that a given result reflects a true effect. We now turn to discuss concrete tools that researchers can use to assess the likely statistical power and Type I error rate of a given test, so that they can calibrate their confidence in the result of that test accordingly.

\section{Tools for Calibrating Confidence in a Study Result}

\section{Assessing Likely Statistical Power}

When planning a study, it is important to think through (a) the intended goal(s) of the study, (b) which statistical test(s) will be focal, and (c) the likely statistical power of those focal tests. With respect to study goals, social cognition researchers are often primarily interested in testing whether two or more conditions differ from each other (e.g., can setting an implementation intention reduce Black/White racial bias in the Shooter Task; Mendoza, Gollwitzer, \& Amodio, 2010). Researchers may also or instead have the goal of testing whether two or more conditions are the same (equivalence testing; Lakens, 2017) or estimating the size of an effect. Importantly, the sample sizes required for a statistical test to meaningfully inform these different goals can be quite different: For example, the sample size needed to estimate the size of an effect with a reasonable degree of precision is substantially larger than the sample size needed to test the existence or direction of that same effect (see Anderson et al., 2017; Schönbrodt \& Perugini, 2013).

Next, it can be useful to specify which statistical test or tests are focal to the goals of the study. For example, social cognition researchers often conduct factorial ANOVAs, but the statistical power to detect a main effect can be dramatically different from the statistical power to detect an interaction. Thus, a given study could be well powered to detect some of the effects in a multi-way ANOVA and severely underpowered to detect others (e.g., main effect tests could be 
relatively informative, whereas a test of the three-way interaction could be quite uninformative). It is therefore preferable to talk about the statistical power of a particular test rather than the statistical power of an overall study, since a study often involves multiple (and differently powered) tests.

After specifying the focal test(s), researchers can estimate the likely statistical power of those tests. This is important because if we ignore statistical power when designing a study, it is easy to end up with underpowered tests that provide poor or inconclusive estimates of the effects or fail to detect them altogether. There are both informal and formal ways to consider statistical power. Informally, researchers can develop and use their intuitions about the sample sizes required to study different kinds of effects (see da Silva Frost \& Ledgerwood, 2020, for a tutorial). For example, the sample sizes required to detect a between-group difference of $d=.20$, $\mathrm{d}=.50$, and $\mathrm{d}=.80$ with $80 \%$ power are approximately $\mathrm{N}=800, \mathrm{~N}=130$, and $\mathrm{N}=50$, respectively. The sample size required to detect an interaction can be much higher and depends on the expected shape of the interaction: For a perfect cross-over interaction, the sample size would be the same; for a knockout interaction, it would be about four times as large; and for a $50 \%$ attenuation interaction, it would be about 14 times as large (see da Silva Frost \& Ledgerwood, 2020, Table 1).

Notice what this means: Most social cognition researchers do not have the resources to conduct meaningfully informative tests of whether small-to-medium effects are partially attenuated by a moderating variable. For example, given that a preregistered study with a large sample estimated the size of the effect of temporal distance on abstraction to be about $d=.276$, 95\% CI [.097, .455] (Sánchez, Coleman, \& Ledgerwood, 2021), a researcher interested in studying whether distraction reduces the size of that effect should be concerned about whether 
they can collect a sufficiently large sample to meaningfully address their question. Detecting a mean difference of $d=.276$ with $80 \%$ power requires a sample size of about $\mathrm{N}=416$, so detecting even a knockout interaction with $80 \%$ power in a 2 x 2 design would require a sample of about $\mathrm{N}=1,664$ (and detecting a partial attenuation pattern would require an even larger sample size). The point here is not that small effects are unimportant, but that small effects (and moderation and mediation of small effects) can be challenging to study in an informative way. By recognizing these challenges, researchers can make informed decisions about how to spend their resources (e.g., is this question important enough to you to spend these resources on answering it?) and how to collaboratively address challenging questions (e.g., a multi-site collaboration to test whether an intervention can effectively reduce a small-to-medium effect in a large, pooled sample).

A Priori Power Analysis. Researchers can also conduct more formal power analyses to get a sense of the likely power of a planned statistical test. For example, when planning a study to test the existence or direction of an effect, researchers can use an effect size estimate that comes from outside the study (e.g., a prior large study or meta-analysis on a similar effect) to calculate the sample size needed to achieve a desired level of power (often $80 \%$ ) for a particular statistical test (e.g., a correlation) given a desired alpha level (typically $\mathrm{p}<.05)$. This type of power analysis is called an a priori power analysis because it is often useful to do before a study is conducted (although it can also be informative after a study is conducted; just be sure not to calculate the power of a test using an effect size estimate from that same test, which is sometimes called "observed power" or "post-hoc power" and which can be very misleading; Gelman \& Carlin, 2014; Hoenig \& Heisey, 2001). A priori power calculations can be computed for many types of statistical tests using software such as G*Power, JAMOVI, SPSS, and R; more 
advanced apps or simulation methods can be helpful for calculating power to detect parameter estimates in structural equation models or multilevel models (e.g., Lane \& Hennes, 2017; Wang \& Rhemtulla, 2020). Researchers can also conduct a priori power analyses that account for stimuli as a source of random variation in the data (e.g., for designs where each participant responds to multiple faces or items drawn from a larger population of stimuli), which can sometimes have a large impact on the power of study designs. When both participants and stimuli are sources of random variation, ignoring the impact of stimuli on power can lead studies to be dramatically underpowered. In some scenarios, low power due to a limited number of stimuli cannot be compensated by even an infinitely large sample size of participants (see Judd et al., 2012, 2017; Westfall et al., 2014).

Sensitivity Power Analysis. Other types of power analysis use the same basic equation relating statistical power to sample size and effect size, but change which two quantities are inputted to calculate the third. In a sensitivity power analysis, a researcher inputs the actual sample size of the study (e.g., $\mathrm{N}=200$ ), the statistical test of interest (e.g., a $t$-test comparing two between-subjects conditions) and a given level of power (e.g., 90\%), and the program outputs the effect size the study was capable of detecting with this level of power (Cohen, 1998; Erdfelder, Faul \& Buchner, 2005). A sensitivity analysis can be useful when researchers want to assess the likely power of a study that has already been conducted, such as a dataset they have already collected or a study in the literature. Even when researchers have already conducted a power analysis when planning their sample size, it can be useful to conduct a sensitivity analysis on the actual sample size when the planned and actual sample sizes are substantially different (e.g., planned $\mathrm{N}=500$ but actual $\mathrm{N}$ after excluding those who failed the attention check $=410$ ). 
A sensitivity power analysis is also useful for study planning when a researcher has no idea what effect size to expect or has a maximum feasible sample size (e.g., a finite participant pool or set amount of money that can be spent on a study), in order to assess the minimum effect size that the planned study will be adequately powered to detect. Sometimes, such calculations can lead researchers to realize that a given sample size is simply insufficient for studying a research question in an informative way (e.g., "This maximum feasible sample size will only give us adequate power to test $d=.5$, but the effect we want to study is probably closer to $d=$ $.25 ”)$, which in our experience is much nicer to know before spending time and resources on a study rather than after.

\section{Assessing Likely Type I Error}

Researchers often assume that the Type I error rate of a test is equal to the alpha cut-off value against which they compare the test's $p$-value (typically, $p<.05$ ). In reality, however, the Type I error rate of a test can inflate (sometimes dramatically) above the nominal alpha level of that test when researcher decisions about how to construct their dataset and/or analyze the results are informed by the data (often called data-dependent or exploratory rather than dataindependent or confirmatory decisions; see da Silva Frost \& Ledgerwood, 2020). When researchers want to conduct an analysis with a particular Type I error rate (e.g., .05), they need to make decisions about sample size, exclusions, covariates, which variables to focus on, and planned analyses independently from the data themselves (Gelman \& Loken, 2014).

There are many contexts in which it is impossible not to know something about one's data (e.g., when conducting analyses on a large, pre-existing dataset when one has already examined some of the variables in that dataset or read other papers based on that dataset), and many contexts in which a more bottom-up, data-driven approach to research is preferable (e.g., 
when conducting the first study in a new line of work, if one is not sure how the participants will respond or simply wants to learn as much as possible from the data without imposing any particular assumptions or expectations onto it). Data-dependent analyses can be extremely useful; it is simply important to know and transparently label when analyses are data-dependent so that researchers can calibrate their confidence accordingly.

A useful tool for clearly distinguishing between data-independent and data-dependent analyses is a carefully constructed pre-analysis plan, which is one particular kind of preregistration (for a quick review of the different kinds of preregistration and the functions they can serve, see da Silva Frost \& Ledgerwood, 2020, Table 4). In a pre-analysis plan, researchers specify ahead of time (before knowing anything about a given dataset) all the decisions that they will need to make about constructing and analyzing a given dataset. These decisions often include planned sample size and stopping rule, inclusion and exclusion criteria, manipulations/conditions, any planned predictors or covariates and how they will be constructed, the dependent measures and how they will be constructed, planned statistical tests, and any plans for correcting for multiple comparisons (e.g., if the planned analyses involve a factorial ANOVA or multiple pairwise comparisons). ${ }^{2}$

Note that pre-analysis plans need not include directional predictions: The Type I error rate of a test is not affected by whether a person has a particular prediction about how the results will turn out (Ledgerwood, 2018). Thus, pre-analysis plans can be useful for distinguishing between data-independent and data-dependent analyses, and for constraining unintentional Type

\footnotetext{
${ }^{2}$ Note that the very common practice of conducting a $2 \times 2$ ANOVA and reporting both main effects and the interaction has a Type I error rate of about $14 \%$ rather than the 5\% that researchers typically assume (see Cramer et al., 2016). One option is to specify ahead of time in a pre-analysis plan which effect is the focal test of interest (e.g., perhaps a researcher is focally interested in the interaction and wants to interpret that $p$-value with high confidence; this can be specified in the pre-analysis plan). Another option is to correct for multiple comparisons so that the entire family of tests (the two main effects and the interaction) has a Type I error rate of .05.
} 
I error inflation, even when researchers are testing exploratory research questions (e.g., "Will my manipulation affect my dependent measure?" or "Theory A predicts one pattern of results and Theory B predicts another...I wonder which one will happen?"). For an accessible tutorial on how to craft an effective pre-analysis plan, see da Silva Frost and Ledgerwood (2020). Table 1 provides a summary of tools that researchers, reviewers, and editors can use to calibrate confidence in a statistical test result by assessing its likely statistical power and Type I error rate. 


\section{Table 1}

Summary of Recommendations for Calibrating Confidence

As a researcher planning a study:

1) Think through the intended goal(s) of the study, which statistical test(s) will be focal, and the likely statistical power of those focal tests

2) Recognize that small effects can be challenging to study in an informative way, and plan accordingly

3) If you have an approximate effect size estimate, conduct an a priori power analysis to calculate the sample size needed for desired power

4) If you have no idea what effect size to expect or a maximum feasible sample size, conduct a sensitivity power analysis

5) Clearly distinguish between data-independent and data-dependent tests by specifying ahead of time any planned, data-independent analyses in a carefully constructed pre-analysis plan (see: da Silva Frost \& Ledgerwood, 2020)

As a researcher writing a paper:

1) Distinguish clearly between planned (data-independent) and unplanned (datadependent) decisions when describing the methods and results

2) Calibrate conclusions to the strength of the evidence (e.g., higher confidence when statistical power is high and Type I error is constrained; lower confidence/more tentative conclusions when power is lower and/or Type I error is not well constrained)

As a reviewer or editor:

1) Request sensitivity power analyses with clear benchmarks for interpreting effect sizes (see: https://www.spsp.org/publications/guidelines-resources\#3)

2) Ask authors to transparently label data-dependent analyses and to calibrate their conclusions to the strength of the evidence

3) Explicitly value transparently labeled analyses, messy results, and appropriately calibrated conclusions (including tentative conclusions)

\section{Part II: Who Are We (Not) Studying? Calibrating the Scope of Our Conclusions}

In Part I, we discussed one particular way in which researchers can calibrate their conclusions to the evidence provided by a study. By considering the likely statistical power and Type I error of a particular statistical test, researchers can adjust their confidence that a 
significant finding reflects a true effect rather than random fluctuation. Of course, there are many other ways in which researchers can and should calibrate their conclusions to a study, including assessing construct validity in order to calibrate their confidence in the meaning of a particular finding (Brewer \& Crano, 2014; Crandall \& Sherman, 2016; Ledgerwood, 2019). In this section, we discuss another important question to ask when assessing a research finding: Whose psychology does it capture?

Scientists studying social cognition are interested in building a systematic understanding of how people think about people. Yet this seemingly straightforward goal is complicated by the historical context of our discipline, which was not developed to be about or for people in general but rather a very narrow subset of people. We will briefly review this historical context and the biases that grew out of it, and then provide some concrete tools for (a) calibrating one's sense of the generalizability of a finding and (b) expanding generalizability when desired.

\section{History and Defaults in Social Cognition}

For the purposes of this chapter, we will focus on the historical context of psychological science as practiced in the United States, but we note that these themes are likely to apply across other geographical areas that share similar historical contexts (e.g., Canada, Australia, and Western Europe) and to professional cultures and scientific institutions that grew out of these contexts. Psychological science, as practiced in the U.S., was not originally set up to be for and about all people — instead, it was set up to be by, for, and about White, cis, straight, wealthy men from European cultures (Keith, 2018). These men developed a scientific and professional culture that worked well for them: one that prioritized neoliberal individual values like individual freedom and identity neutrality, and one that focused on understanding the psychology of White men like themselves (Cheryan \& Markus, 2020; Ledgerwood et al., 2021; Salter \& Adams, 2013; 
Thalmeyer, Toscanelli, \& Arnett, 2020). This culture permeates our institutional norms, values, and assumptions; without intentional intervention, defaults and assumptions often revert to that historical pattern (McCormick-Huhn et al., 2019).

For instance, the vast majority of psychological studies involve primarily White, Englishspeaking participants from so-called "Western" countries (i.e., countries in the global northwest; Henrich, Heine, \& Norenzayan, 2010; Thalmeyer, Toscanelli, \& Arnett, 2020). Meanwhile, scholars (and especially White scholars) often assume that the psychology of these participants generalizes to all humans (Roberts, Bareket-Shavit, Dollins, Goldie, \& Mortenson, 2020). This assumption no doubt reflects the same biases documented by social-cognitive researchers for people in the U.S. to think of other people by default as being male and to think of Americans as White (Bailey, LaFrance, \& Dovidio, 2020; Devos \& Banaji, 2005; Yogeeswaran \& Dasgupta, 2010; see Remedios \& Vinluan, this volume, for a deeper discussion). For example, a recent analysis found that psychology paper titles were more likely to specify sample characteristics (implying constraints on generalizability) when the research involved participants from outside the United States or focused on non-White U.S. participants—in other words, authors were more likely to use titles implying generality when studying White participants in the U.S. than when studying non-White participants and participants in other countries (Cheon, Melani, \& Hong, 2020).

Like many biases, when left unnamed and unexamined, these biases can lead us astray. For example, Oosterhof and Todorov's (2008) valence-dominance model of face evaluation sought to describe how "people automatically evaluate faces" (p. 11087) by examining how presumably U.S. participants (race and country were unreported) evaluate White faces. The conclusions they drew about "the underlying dimensions of face evaluation" (p. 11087) and the 
evolutionary bases for these dimensions were easy to read as describing the underlying dimensions of how people in general evaluate faces in general, rather than specifically describing how people in their sample evaluated White faces. However, recent collaborative research across 41 countries in 11 world regions revealed evidence for regional differences in face evaluation, suggesting that the original results do not generalize as far as default biases would lead one to assume (Jones et al., 2021). In other words, the scope of the conclusions that many social cognition researchers drew from Oosterhof and Todorov (2008) was miscalibrated: We assumed that the model generalized further than the evidence actually suggested.

Indeed, there is considerable evidence to suggest that the psychology of English-speaking White participants in Western countries does not generalize to the global majority (e.g., Majid et al., 2004; Heinrich et. al., 2010; Song et al., 2020; Tropp \& Wright, 2003). As just one example, people in the United States are unusually and extremely individualistic relative to people in the rest of the world (Heinrich et al., 2010), and this unusual individualism is particularly characteristic of White Americans (Vandello \& Cohen, 1999). Evidence against generalizability has been found even for cognitive tasks that are sometimes assumed to tap processes that are so "basic" or "low-level" that they must generalize. For example, research has documented high variability across populations in visual illusions, such as the Muller-Lyer optical illusion task in which two lines of equal length appear different to participants in some cultures, and U.S. undergraduates were at an extreme end of the distribution (Heirich et. al., 2010; Segall et. al., 1966). Another example can be found in spatial cognition: Even though egocentric frames of reference (using the self as a reference point) were long assumed to be innate concepts, in a comparison of 20 languages of diverse societies, only three used an egocentric frame as the 
preferred system of reference - and they were all from industrialized populations (Majid et. al., 2004).

\section{Tools for Calibrating Generalization: Delineating the Borders of a Research Finding}

Given the biases described above, it is important for researchers to explicitly describe who they are and are not studying; in the absence of explicit statements, people (and especially dominant group members) may be likely to miscalibrate the scope of conclusions that they draw from a given paper or body of research. In this section, we describe research practices to better calibrate the scope of a study's conclusions.

First, incorporate descriptive information about participant and stimulus samples into article abstracts. For example, a study examining trait inferences about White faces among mostly White participants or motivated partner perceptions in mixed-gender couples should clearly state this focus in the abstract. Indeed, some journals are beginning to require that authors report basic demographic information about study participants in the abstract of their manuscripts (e.g., Child Development, 2020). Using an intersectional lens to inform such descriptions can help avoid the common mistake of conflating an entire group with its most privileged members (e.g., describing research about White women as research about women in general, or research about evaluations of Black vs. White men's faces as research about evaluations of Black and White people in general; see McCormick-Huhn et al., 2019; PurdieVaughns \& Eibach, 2008; Remedios \& Vinluan, this volume). Clearly demarcating the breadth of participant and stimulus samples at the beginning of a paper helps both authors and readers calibrate their understanding of a paper's generalizability.

Second, explicitly report participant demographics, including race/ethnicity and national context, when describing participants in the method section. Despite considerable research 
demonstrating the importance of racialized experiences in shaping a wide range of psychological processes (Roberts \& Rizzo, 2020), a recent analysis found that the vast majority of psychology articles fail to mention racial information about participants, often while describing results with generic language implying that the findings generalize to all humans (DeJesus et al., 2019). When collecting demographic data, follow up-to-date recommendations for inclusive practices (for example, inform participants about the purpose of collecting demographic information and use inclusive language; see e.g., Puckett et al., 2020). When examining demographic data, focus on describing the sample or understanding variables that can explain demographic differences in results; it is rarely advisable to use demographic categories themselves as explanatory constructs (Helms et al., 2005). That is, researchers should avoid the pitfall of treating demographic characteristics (e.g., race) as essentialist and reflective of innate attributes, or using a researcher's own ideas about groups to fill in the explanatory gap. Instead, it is important to unpack such results to understand how, when, or why demographically distinct groups show different patterns of social cognition. Doing so provides more nuance and complexity in understanding how people think about other people, uncovering neglected components of the metaphorical sculpture.

Third, include a Constraints on Generality statement in the General Discussion of each paper (Simons, Shoda, \& Lindsay, 2017). Such a statement explicitly describes the target populations from which participants and study materials were sampled and clarifies the authors' intended scope of generality. For example, a researcher studying how mostly White U.S. undergraduate participants evaluate White faces from the Chicago Face Database (Ma et al., 2015) might expect their results to generalize very narrowly (to very similar participants and targets), very broadly (to all humans), or somewhere in between. A Constraints on Generality statement clarifies the intended scope of generalization; writing one can also be a very useful 
tool for helping authors avoid inappropriately generic language in the rest of the manuscript (see DeJesus et al., 2019).

Fourth, when reviewing past research in a review paper, chapter, or empirical paper introduction, choose language that carefully calibrates the scope of the claims to the actual samples included in the research (Rogoff, 1998). For example, authors can clearly indicate the communities in which past research took place (e.g., "research conducted in college samples in the United States and Canada has found...”). Authors can also describe findings in terms of "observations that have occurred with particular participants in research ('these children did such-and-such') rather than in general form ('children do such-and-such')" (Rogoff, 1998, p. 723).

Finally, it is important to attend carefully and consistently to these issues in our roles as reviewers and editors. The temptation to use generic language may stem from a tendency to perceive generically worded findings as more important (DeJesus et al., 2019). Reviewers and editors can play an important role in pushing back against these tendencies by asking authors to include descriptive information about participants and stimuli, in the four ways described above, and to calibrate their conclusions accordingly. When these elements are present in a paper, reviewers and editors can explicitly emphasize and value their importance when writing reviews or decision letters. Reviewers and editors should also take care to place similar value on narrow samples that focus on more dominant or marginalized groups: For example, if a journal routinely values and publishes research on primarily White U.S. undergraduates or online workers, reviewers and editors should similarly value and support publishing research on primarily Black U.S. undergraduates or primarily Middle Eastern workers. Avoid characterizing the latter 
examples as more "specialized" or less "basic" (which would be inaccurate and racist, as the above discussion makes clear).

Table 2 provides a summary of tools that researchers, reviewers, and editors can use to help calibrate the scope of a study's conclusions.

\section{Table 2}

Summary of Recommendations for Calibrating Generalization

As a researcher writing a paper:

1) Incorporate descriptive information about participant and stimulus samples into article abstracts.

2) Explicitly report participant demographics, including race/ethnicity and nationality, in methods sections.

3) Include a Constraints on Generality statement in the General Discussion

4) Clearly indicate the communities of focus when reviewing past research.

5) Describe findings in appropriately specific terms (e.g., "these participants did X" instead of "people do X").

As reviewers and editors:

1) Explicitly request and value the importance of the five points above when writing reviews and decision letters.

2) Value and support research that focuses on marginalized groups instead of considering it as "specialized" or less "basic."

3) Include these expectations in guidelines for authors and reviewers

\section{Tools for Expanding Generalizability}

In addition to better calibrating the scope of our inferences to the existing evidence, we can also take steps to diversify our samples and stimuli within a study or across studies. For example, researchers can use stimulus sets that draw from diverse populations (e.g., for faces, the American Multiracial Faces Database and the Chicago Face Database; Chen et al., 2020; Ma et al., 2015, 2020). When researchers want to make a claim about how participants respond to a 
given population of stimuli (e.g., job applications from White versus Black candidates), it is crucial to sample multiple, representative stimuli from the population of interest (e.g., job applications paired with several different White and Black faces). When possible, consider repeated measures designs in which each participant encounters multiple stimuli (e.g., faces, vignettes, or scenarios) and consider randomly assigning participants to view a subset of the total available stimuli. When planning the study and analyses, plan to account statistically for stimulus sampling to allow generalization (see Judd et al., 2012) and power the study accordingly (note that the number of stimuli can be more important for power than the number of participants; see Westfall et al., 2014).

Methods for identifying appropriate culturally and ethnically diverse samples have been well articulated (Leong et al., 2010; Matsumoto \& van de Vijver, 2010). To investigate whether a process is universal, for example, it is ideal to include comparative samples that differ on as many attributes as possible, whereas to tie an identified group difference to a contextuallyderived attribute or process, it is ideal to select samples that are similar in many ways, but differ on the attribute of concern. Once samples are identified, it is important to employ methods that are mindful of potential bias or assumptions of methodological or psychological equivalence. Researchers must resist a colonial approach to psychological science that assumes the methods and observations developed in one particular context can be unthinkingly applied to another (see also de Vos, 2020; Thalmeyer et al., 2020). Thus, seeking out and including emic knowledge and methodological approaches is important (see Adams, 2005).

To that end, it is often useful to work collaboratively with other researchers who have theoretical and experiential knowledge of and access to different participant populations. Collaborations can be built informally by connecting through social media or at conferences, 
searching for published research — from a broad range of journals — that includes samples from the desired regions, or exploring universities or other institutions located in regions of interest. They can also be built more formally, through mechanisms such as the Psychological Science Accelerator (an initiative that coordinates data collection for selected studies across a global network of laboratories; Moshontz et al., 2018). Prioritizing such connections can also be useful for building collaborative networks that can bring more diverse perspectives to guide future research projects (a topic we discuss more fully in Part III).

Large-scale cross-cultural projects or replications reflecting such collaborative networks provide one way to quickly incorporate diverse researchers and samples, and such projects can be valuable in establishing variations in effect size across samples and settings (Klein et al., 2018). However, in selecting effects to explore, these projects often employ an etic perspective, looking at how an established effect generalizes, thereby shining light on only part of the sculpture-one which is already well-lit, front and center.

When embarking on cross-cultural or cross-group replications of effects generated in White and Western culture, researchers often use the experimental stimuli or scales initially generated in a single, White and Western cultural context. This top-down process can sometimes be useful (e.g., when testing whether the psychometric properties of a scale are similar across cultures), but also makes it difficult to ensure that materials have similar psychological significance across multiple groups. For this reason, it is important to complement such research with work that employs emic knowledge from the beginning (Berry, 1999; Morris et al., 1999). Thus, when designing new and targeted studies, we echo cultural psychologists before us (see Adams, 2005; Hruschka et al., 2018) to recommend adopting an inclusive and bottom-up approach: Researchers familiar with each cultural context or group of interest can work 
collaboratively to select effects of interest, whether they have been investigated previously or represent new perspectives, and design appropriate methods for studying them. Simply translating existing materials to other languages without considering the psychological meaning in each cultural context undermines researchers' abilities to draw conclusions about psychological generalizability or specificity.

More broadly, in order to be informative, cross-cultural research needs to consider multiple kinds of equivalence: not only linguistic equivalence (meaning that materials are translated accurately) but also metric equivalence (the psychometric properties are the same), conceptual equivalence (a common set of behaviors define a construct), and functional equivalence (two or more behaviors are functionally related to similar problems; see Leong et al., 2010). Meanwhile, editors and reviewers should explicitly value and prioritize such work when submitted by research teams from underrepresented groups or countries, even or perhaps especially when methods diverge from those dominating in the United States, Canada, Western Europe, Australia, and New Zealand. Embracing such novel methods, samples, and research teams is critical to illuminating psychological effects that otherwise live in the shadows. For a summary of tools for expanding generalizability within or across studies, see Table 3. 


\section{Table 3}

Summary of Recommendations for Expanding Generalizability

As a researcher planning a study:

1) Use stimulus sets that draw from diverse populations

2) Sample multiple, representative stimuli from the population of interest

3) Account for stimulus sampling in power calculations and statistical analyses

4) Conduct thoughtful cross-cultural research that resists a colonial approach by seeking out and including emic knowledge and methodological approaches from the beginning

As a reviewer or editor:

1) Explicitly recommend and value the importance of the four points above when writing reviews and decision letters

2) Value and prioritize work submitted by research teams from underrepresented groups and countries, even or especially when methods diverge from dominant approaches

\section{Part III: Whose Perspectives are Missing? Cultivating Diverse Positions of Inquiry}

To that end, in order to build a comprehensive understanding of how people think about people, social cognition researchers need not only to study diverse samples but also to ask questions from diverse vantage points. Returning to the example at the opening of this chapter: If we want to understand the sculpture in the middle of the dark room, we cannot just examine it from one particular person's location. Instead, we develop a comprehensive understanding by examining the sculpture from multiple perspectives. In our science, we develop a comprehensive understanding by including a diverse array of researchers who can ask questions, design studies, and interpret results in a way that is informed by a wide range of identities and experiences.

However, as described in the previous section, our discipline (like higher education more broadly in the U.S. and similar countries) was not set up to cultivate and support diverse positions of inquiry (Bulhan, 2015; Guthrie, 1976; Keith, 2018; Kendi, 2017). Rather, it was set up to cultivate and support one specific and very narrow perspective on mind and behavior: that 
of wealthy, White, cisgender, straight, men living in a small subset of countries. Our institutions are set up to work well for people who are similar to those who created those institutions, but not for everyone else; indeed, they work less and less well for people whose identities and experiences place them further from the included center (Ledgerwood et al., 2021).

This exclusive system impedes our shared goal of advancing cumulative knowledge about mind and behavior. Scientific understanding is shaped by researchers and institutions. The perspectives, choices, and biases that enter into the process are manifested in the outcome. As Alondra Nelson, the U.S. Deputy Director for Science and Society, recently stated: "When we...design, test, and research, we are making human choices, choices that bring our social world to bear in a new and powerful way. It matters who makes these choices. It matters who they're thinking about when they do" (Nelson, 2021). Historically, a narrow subset of voices has been augmented in our science while others have been repressed. Consequently, our understanding of social cognition (like many other topics) reflects a dominant and incomplete picture - a snapshot of the sculpture taken from one particular location in the room, missing many other perspectives.

\section{Researchers' Experiences and Positions Inform Science}

Researchers studying the science of social cognition are both helped and hindered by our disciplinary lens in understanding the importance of positionality and experience. On the one hand, as social cognition researchers, we are intimately familiar with research demonstrating the various ways in which people's expectations, identities, and prior knowledge influence the questions they choose to ask, the types of information they seek out, and how they process and draw conclusions from that information (e.g., Chaiken \& Ledgerwood, 2012; Danbold \& Unzueta, 2020; Higgins \& Rholes, 1976; Klayman \& Ha, 1987; Skov \& Sherman, 1986; Dawson, Gilovich \& Regan, 2002; Brewer \& Nakamura, 1984; Hirt, MacDonald, \& Erikson, 
1995). On the other hand, our broader scientific culture of neoliberal individualism (Cheryan \& Markus, 2020; Salter \& Adams, 2013) emphasizes the value of identity-neutrality, which can be difficult to reconcile with the notion that researchers' identities and experiences shape their research. Scholars can be left in the somewhat ironic position of recognizing the process in the abstract but failing to admit that it applies to ourselves.

And yet, once we recognize that identity and lived experiences can shape and inform the questions we ask and the answers we get, we can appreciate how much stronger science can be when it includes diverse positions and perspectives (Garay \& Remedios, in press). For example, topics that historically have been studied by researchers whose identities and experiences place them close to the included center of the field (e.g., affluent, White, straight, cis men) can benefit from non-dominant perspectives that contribute to a theoretical understanding of various socialcognitive phenomena.

Indeed, several groundbreaking ideas in social psychology were developed and refined by non-dominant researchers who leveraged their unique vantage points into empirical questions and novel hypotheses. One example is Black male researcher Claude Steele. In the early 1990s, Steele was tasked with investigating why Black students in good standing with excellent SAT scores at the University of Michigan had a much higher drop-out rate compared to White students. Steele identified several patterns that led him to examine the impact of negative stereotypes on Black students' academic performance and to develop the concept of stereotype threat, which captures the experience of threat that stereotyped group members can have when they are at risk of potentially confirming a negative stereotype about their own group (Steele, 1999; Steele \& Aronson, 1995). The concept of stereotype threat focused scientific attention on the social psychology of stereotyping from the point of view of those who are negatively 
stereotyped, enabling a more complete picture of the functioning and consequences of stereotypes (see e.g., Lewis \& Sekaquaptewa, 2016).

Another example is the work of Brenda Major and Jennifer Crocker: two White women who studied why members of stigmatized groups have lower self-esteem than members of nonstigmatized groups. Their work uncovered that women who received negative feedback from a prejudiced evaluator will attribute that feedback to prejudice, and not their own behavior, thus lowering their rates of depression compared to women who received negative feedback from a non-prejudiced evaluator. This discovery, informed by their own experiences (Society for Personality and Social Psychology, 2018), led to attributional ambiguity theory, which states that members of stigmatized groups attempt to preserve self-esteem by attributing negative feedback to prejudice (Crocker, Voelk1, Testa \& Major, 1991).

In both of these cases, researchers advanced social psychology by identifying and studying the psychological experiences of members of a marginalized group to which they themselves belonged. Their specific identities and experiences positioned them to approach the metaphorical sculpture at the center of the room from a particular vantage point, illuminating an area that may have been difficult for dominant group members to notice or understand from their own perspective. Diverse perspectives enable a more comprehensive understanding of social psychology than any one perspective can accomplish on its own. ${ }^{3}$

\section{Narrow Perspectives Restrict Scientific Understanding}

However, because our discipline was set up to cater to a narrow subset of people, it tends to exclude and devalue perspectives that depart from the dominant viewpoint (Ledgerwood et al.,

\footnotetext{
${ }^{3}$ While these perspectives are not free of controversy (e.g., Forscher et al, 2020), the point is that they might not ever have been a part of the conversation without researchers asking questions about social psychology from a range of vantage points.
} 
2021). As a result, we are not well positioned collectively to encircle the metaphorical sculpture and understand it from all sides. Instead, our collective view is a relatively narrow one, restricting our scientific understanding and continually pushing non-dominant scholars and topics to the margins of the field.

For example, although White people are a global minority, most psychological scientists in the field's dominant organizations are White (e.g., APS, 2018). As research from our own discipline makes clear, race and racism play major roles in shaping mental processes and behavior (e.g., Dovidio, Gaertner \& Pearson, 2017; Rivera et al., 2021; Sawyer, Major, Casad, Townsend \& Mendes, 2012). As just one illustration, recent evidence suggests that White faces may be processed differently from other faces (Xie, Flake, Stolier, Freeman, \& Hehman, 2021). However, despite the central importance of race and racism for structuring psychological experience, White people are especially unlikely to notice or talk about race and racism (Nelson et al., 2012; Pauker et al., 2015; Perry et al., 2019). White psychologists are no exception: The vast majority of psychological research articles do not mention race (DeJesus et al., 2019), and White editors are less likely to publish papers that highlight race (Roberts et al., 2020). Thus, because of the narrow (predominantly White) vantage point currently represented in our field, we may be missing key elements of how race and racism infuse many aspects of mental processes and behavior (e.g., Salter et al., 2017).

Even when White researchers directly study race and racism, they often overlook important features (like hierarchical systems of oppression) that tend to be much more obvious to those experiencing racial oppression first-hand (see Roberts \& Rizzo, 2020, for a review of research on the psychology of American racism). In contrast, scholars of color can often draw on 
a lifetime of structural inequality experience that well equips them to identify the flaws woven into the system. Those marginalized by a given system are often best positioned to perceive it. More broadly, research questions in social psychology tend to be framed, prioritized, and pursued from the dominant perspective. As noted earlier, researchers have often assumed that White participants from Western societies represent standard or prototypical participants and that phenomena observed in such samples are universal (Henrich et al., 2010; Purdie-Vaughns \& Eibach, 2008; Remedios \& Snyder, 2015). There is an inherent disadvantage in research that uses any one particular group as the sole starting point and standard, which has been dubbed the home-field disadvantage (Medin, Bennis \& Chandler, 2010). When one particular group is used as the starting point for a line of inquiry, it can bias the kinds of questions that researchers think to ask and the answers that they find.

Likewise, when researchers start with the assumption that measures developed for one particular and familiar context can be applied to other contexts, it can contort the information provided by a study. For example, the Likert-type scale (Likert, 1932) is widely employed by scholars as a reliable and effective strategy to collect data on subjective judgments. However, the meaningfulness of data collected using Likert-type scales may be limited to well-studied populations (Hruschka, Munira, Jesmin, Hackman \& Tiokhin, 2018). Respondents in the global northwest (e.g., the U.S., Canada, and Western Europe) who have years of experience with Likert-type measures may find this tool to be a quick and intuitive method to provide their judgements. However, this perspective is not consistently observed in all populations (e.g., Flaskerud, 2012, Winking et al., 2018). When one research team attempted to collect Likert-style data from a group of Southeast Asian respondents, about half of them expressed difficulty understanding this format (Flaskerud, 2012). Rather than rating their feelings by selecting a 
single option from “always," "often," "sometimes," "rarely," and "never," respondents preferred to answer "yes" or "no" to each option. The researcher would have to ask "Do you always feel sad", "Do you often feel sad", "Do you sometimes feel sad," etc., to which the respondent answered yes or no. A similar difficulty in interpreting a continuum of responses in favor of discrete and dichotomous responses was observed in a group of Salvadoran refugees in Central America (Flaskerud, 2012). Thus, even the field's most basic default assumptions, such as the common methodological assumption that meaningful data can be collected using Likert-type scales, have developed in a very particular historical and geographical context that centers dominant group members' perspectives and methods. When a research team does not include perspectives from outside this narrow context, they can easily miss how their default assumptions limit their thinking and their research.

\section{Tools for Increasing Diversity and Inclusion in Social Cognition Research}

As we consider strategies to move closer toward our goals of greater equity, diversity, and inclusion in how we approach the study of social cognition, we must consider our starting point as a field. As argued above, the field (like many others) has been shaped by its history. It was set up to prioritize the values and needs of a narrow subset of people, and without careful and sustained intervention, it will continue to prioritize this included center while deprioritizing everyone else (and especially those whose identities place them farther from rather than closer to that center). Simply put, our field has too few scholars who can approach research from historically excluded vantage points. Moreover, our field tends to undervalue and contort such perspectives by requiring that researchers frame their inquiries from the perspective of the status quo (Ayoub \& Rose, 2016). Thus, if we want to be able to understand the sculpture at the center of the room by approaching it from a variety of vantage points - and if we recognize that the 
subject matter belongs to everyone-we have work to do. In this section, we offer some concrete tools for doing this work (see Ledgerwood et al., 2021 for a deeper discussion).

Simply recognizing the value of new perspectives provides a useful starting point for advancing diversity and inclusion in research. Researchers should complement their existing perspectives by working to build diverse and inclusive research teams (Ledgerwood, Pickett, Navarro, Remedios, \& Lewis, in press), including undergraduate research assistants, graduate students, postdoctoral scholars, faculty, and community partners. Focusing on the value of added perspectives helps guide attention to the importance of ensuring that diverse perspectives are not only present but also included: Do research assistants feel comfortable voicing their ideas during lab meetings and does their feedback shape the work? Are collaborators' ideas valued and incorporated into the research project?

More broadly, it is important to make diversity and inclusion a starting point in one's work rather than an afterthought (González-Márquez \& Tennant, 2021). From a process perspective, it is harder to incorporate a new perspective into an idea once it is fully baked. From an interpersonal perspective, scholars of color may be suspicious of the motives of those who reach out to them for collaborative opportunities, particularly when done at the last minute or in the final stages of preparation. One of the authors of this chapter has been approached on several occasions to join grants in a quasi-scientific capacity in order to help the PIs meet diversityrelated mandates imposed by the granting agency. The perceived motivations underlying invitations to collaborate matter, and suspicion that those motives do not reflect genuine intentions (Kunstman et al., 2016) or a mismatch of goals for the collaboration (Bergseiker, Shelton, \& Richeson, 2010) can prevent or disrupt collaborative efforts. This suggests that efforts to diversify research teams and collaborators directly acknowledge the importance of diversity in 
the formulation and development of a research idea. Potential collaborators should have little doubt as to why they are being approached and how you believe they can benefit the project from a scientific standpoint.

These same considerations are important when engaging in the kind of cross-cultural research described in Part II. Cross-cultural work is better informed when research teams include members with expertise in each culture of interest and when this expertise is appropriately valued and incorporated from the beginning. For example, it is important to have deliberate conversations with team members about the psychological process of interest and methods for exploring it - decentering methodology from any one cultural perspective and ensuring that goals and methods are informed by multiple cultural perspectives. It is also important to engage in deliberative discussions about the research goals, resources, and lived experiences of collaborators and their target samples. Researchers need to consider not only how differences could affect results and conclusions, but also whether they evoke different ethical costs or consequences given that laws and protections of civil liberties vary widely around the world. For example, a researcher in one country might include a variable in a dataset or a term in a paper that is common to use in their own country, without realizing that it could put participants or researchers at risk in another country. When collaborating across cultures and countries, researchers need to include experts from each area in every decision, and be ready to make adjustments or change focus when necessary.

In hosting such deliberate conversations, it is critical to listen to collaborators, and to request that they carefully vet hypotheses and materials rather than simply acquiesce to dominant paradigms and practices. Researchers may also look for ways to share research infrastructure and resources to help ensure that costs and benefits across collaborative groups are equitable. 
Researchers should never assume that the processes, structures, and institutions that support research necessarily work in the same way across countries or contexts. Ensuring adequate support for researchers who have less access to resources, or for whom conducting research amasses hidden burdens, will benefit the discipline as a whole through expanded knowledge and understanding.

As reviewers and editors, scholars should advocate strongly for the importance of topics, paradigms, and methods that have been historically pushed to the margins of the discipline. Start questioning the assumption that certain topics or methods belong in "specialty outlets" or do not "fit" a particular journal or funding mechanism; the question of what is "broadly" interesting or appealing is often a question about what is interesting or appealing to the historically included center of the field (i.e., cis, straight, White men in the U.S., Canada, Western Europe, Australia, and New Zealand). In a review or decision letter, go beyond a sentence saying "It's nice to see the authors using open and inclusive methods" and explain exactly why the methods are valuable and important to publish. Start meeting with other reviewers and/or editors to question assumptions, learn from marginalized vantage points, and develop concrete strategies for enhancing diversity and inclusion in your current editorial role. For example, an editorial team could meet monthly to discuss a recent article, listen to an invited speaker, or make plans for diversifying the editorial board and implementing new editorial practices. Finally, editors can make concrete policy changes that enhance attention to the ways in which a researcher's vantage point informs their research (e.g., by asking authors to include positionality statements in their manuscripts and creating online guidance or concrete recommendations to help authors write effective statements). Table 4 provides a summary of practices that researchers, reviewers, and editors can use to improve diversity and inclusion in social cognition research. 


\section{Table 4}

Summary of Recommendations for Increasing Diversity and Inclusion

As a researcher:

1) Acknowledge the importance of diversity in forming and developing a research idea

2) Build diverse and inclusive research teams that center marginalized voices throughout the research process

3) Ensure adequate support for team members who are less well resourced

As a reviewer or editor:

1) Advocate strongly for the importance of topics, paradigms, and methods that have been historically pushed to the margins of the discipline (rather than suggesting they be published in "specialty outlets" or do not "fit" a journal or funding mechanism)

2) Meet regularly with other reviewers or editors to question assumptions, learn from marginalized vantage points, and develop strategies for enhancing diversity and inclusion in your current editorial role including your editorial board

3) Ask authors to include positionality statements in their papers that describe the assumptions, experiences, and identities that form the lens through which they approach their work

\section{Part IV: How to Make Systemic Changes to Support Open, Replicable, and Inclusive}

\section{Science}

Of course, individual investigators do not operate in a vacuum; they are also influenced by the structures and priorities set by organizations that support and guide their efforts. Systems and policies can influence people, but people create and maintain systems and policies.

Because our incentive systems are interdependent and historically entrenched, it is tempting to think that change must occur "over there" before it can occur "over here," or at a higher level before it can happen at a lower level. And when contemplating such a large, interconnected set of incentive structures, it is easy to feel powerless. But in reality, to improve our science, we need people to intervene in parallel at every level and in whatever roles and contexts they currently occupy. 
It can be helpful first to locate yourself within the system (see Figure 2 for a worksheet that can help you do this, which builds on resources developed by Bellamy et al., 2020). First, what identities do you have, including those that have been historically included as well as historically excluded from academia? What do these identities enable you to perceive clearly and what do they obscure? Where do you experience power or privilege, and where do you experience oppression or exclusion?

Next, what roles, networks, and contexts do you have access to? Consider not only your title (e.g., research assistant, PhD student, post doc, assistant professor) but also the various micro contexts (e.g., lab, classroom, committee, task force) and macro contexts (e.g., department, school/university, journal, society, granting agency) that you occupy and your role within those contexts. Where could you have decision-making power? Where could you have influence? Where could you organize with other people to increase your influence? 
Figure 2

Worksheet for Locating Yourself and Planning Action within Academic Systems

1. What identities do you have (both historically included and excluded from academia). For example: race, gender, sexuality, citizenship, class, language(s) spoken, disability, neurodiversity.

2. What roles, networks, and contexts do you have access to? For example: graduate student, committee member, teaching assistant, instructor, task force chair, talk series organizer, grant panel member, department chair, author, reviewer, editor, social media network, lab, journal club, writing group, blog audience.

For 3 and 4: Sometimes, your power in a particular role or network is as a gatekeeper, sometimes as an advocate, and sometimes both.

- Gatekeeper strategies: Select, implement, reward, incentivize.

- Advocate strategies: Suggest, amplify, organize, persuade, pressure.

3. In what roles or contexts do you have power as a gatekeeper? What specific strategies will you use?

4. In what roles or contexts do you have power as an advocate? What specific strategies will you use? 
Sometimes, your power in a particular role or network is as a gatekeeper or decisionmaker: You have the power to make and execute decisions and policies. For example, a lab manager or graduate student may have the power to decide how to recruit and select research assistants in their lab; a journal editor may have the power to change the submission guidelines for their journal. Sometimes, your power in a particular role or network is as an advocate: You cannot change policy directly or independently, but you can influence gatekeepers who do have that power. In Table 5, we provide recommendations for how to change incentive structures to promote open, replicable, and inclusive science (see Dupree \& Kraus, 2021; Ledgerwood et al., 2021; Stevens et al., 2021 for additional ideas and recommendations). You can pursue these recommendations as a gatekeeper, an advocate, or both, depending on the strategies available to you in a particular role and context.

For example, a person filling out the worksheet provided in Figure 2 might write in the gatekeeper section: 'I'm a gatekeeper as an author, reviewer, associate editor, instructor, and society board member. As an author, I will attend to author diversity when selecting what work to cite. As a reviewer, I will consistently ask authors to include information about participant race/ethnicity and nationality in their abstract, power analyses, a constraints on generality statement, and a positionality statement. As an associate editor, I can select what papers to publish and incentivize open and inclusive science practices through my decision letters. As an instructor, I can select what work to cover in my course and I will audit and work to increase the diversity of the scientists represented in terms of race, gender, and nationality. As a board member, I will propose adding to our society's award criteria new language valuing open, replicable, and inclusive methods and practices. 
In the advocate section, they might write: "I can be an advocate in my role as associate editor. I will suggest ideas for incentivizing open and inclusive practices to the Editor-in-Chief, I will organize with other associate editors to develop, test-drive, and then lobby for a rubric to evaluate open and inclusive practices, and I will enlist the Editor's help in figuring out how to get the journal to adopt an inclusive name change policy. In my roles as a department member and society board member, I will consistently amplify the voices of marginalized group members by emphasizing their comments in meetings (e.g., "I agree with X," "Y raises an important point")."

Whatever your roles happen to be, action is key. Collectively, our individual actions and inactions comprise our systems. All actions and inactions to change our incentive systems are therefore active choices about the kind of science that we want to have. 
Table 5

How to Change Incentive Structures to Promote Open, Replicable, and Inclusive Science

\begin{tabular}{lll}
\hline Decision Sphere & Examples of Influence & Recommendations \\
\hline $\begin{array}{l}\text { Personnel: } \\
\text { Who participates in } \\
\text { science? }\end{array}$ & $\begin{array}{l}\text { Selecting research assistants, } \\
\text { admitting and funding students, } \\
\text { selecting participant samples, hiring } \\
\text { and promoting faculty, writing tenure } \\
\text { letters, selecting editors, nominating } \\
\text { and voting for leaders }\end{array}$ & $\begin{array}{l}\text { Value experiences and skills that enhance inclusive excellence (e.g., cultural } \\
\text { competence, underrepresented perspectives). }\end{array}$ \\
& $\begin{array}{l}\text { Devote resources to supporting the inclusion of underrepresented perspectives } \\
\text { labs with different resource levels) }\end{array}$ \\
& $\begin{array}{l}\text { Value contributions to systems and infrastructure that help good science } \\
\text { flourish (e.g., creating open science resources; building new and inclusive } \\
\text { networks). }\end{array}$ \\
& $\begin{array}{l}\text { Create a transparent rubric that explicitly weighs various types of experiences, } \\
\text { skills, and/or labor essential to open and inclusive science. }\end{array}$ \\
& $\begin{array}{l}\text { Replace bean-counting heuristics (publication counts, impact factor) with } \\
\text { metrics and judgments that assess the extent to which a scholar has adopted } \\
\text { practices that promote well calibrated conclusions and inclusive science. }\end{array}$
\end{tabular}


Research Content:

What work is

published?
Reviewing and editing journal articles, reviewing conference submissions, inviting contributions to journals or books.
Attend to (or develop explicit rubrics for assessing) the openness and inclusiveness of research methods and prioritize these features in your decision.

Require submissions to explicitly address the strengths and limitations of the work in terms of strength of evidence, scope of conclusions, and positionality of authors.

Recognize that any one paper or presentation will have multiple limitations; prioritize work with well calibrated conclusions over work that over-claims.

Develop resources (guides, checklists, trainings) to support authors and reviewers/editors in (1) calibrating confidence in study results, (2) calibrating scope of conclusions, (3) cultivating diverse positions of inquiry.

\section{Dissemination: \\ What work is communicated and discussed?}

Choosing who you cite in your manuscripts, choosing who to follow and what work to promote on social media, choosing who to write for, selecting work to cover in syllabi and reading lists, inviting speakers or panelists
Attend to the diversity of the scholars whose work you are choosing to disseminate.

Value underrepresented vantage points.

Value open, replicable, and inclusive methods and practices.

Increase accessibility by translating abstracts into multiple languages, writing for non-academic audiences, and making scientific content publicly available whenever possible.

\section{Reward: What work is funded and awarded?}

Award nominations, award cristee decisions, creating or revising award criteria, awarding grant funding
Value underrepresented vantage points.

Value open, replicable, and inclusive methods and practices.

Fund and award diverse and inclusive teams rather than individual scholars. 


\section{Conclusion}

In this chapter, we have suggested that scientists wishing to understand social cognition can and should advance their methods and practices in three important ways. First, we reviewed concrete tools that can help researchers appropriately calibrate their confidence in a significant finding to the strength of evidence provided by the finding. Second, we described methods and practices that researchers can use to calibrate how broadly they generalize from a particular finding, as well as tools researchers can use to expand generalizability when they want to do so. Third, we discussed how researchers can increase the range of perspectives included in our field, so that our collective understanding of how people think about people can be informed by multiple vantage points. And finally, we pointed to the importance of taking action at every level to change incentive structures so that they reward the kinds of practices discussed in this chapter.

Together, we hope that the ideas reviewed in this chapter provide researchers with a useful toolkit for advancing a cumulative and comprehensive science of social cognition. Adopting these methods and practices will make our science stronger. But that is not the only reason, or even the most important reason, to do them. The status quo in psychological science reflects the deep inequities inherent in society more broadly (Bowleg, 2021; Bulhan, 2015; Ledgerwood et al., 2021), and our practices contribute to the maintenance or disruption of those inequities. We are, ostensibly, scholars dedicated to understanding human thinking and behavior to the benefit of science and society. Yet our continued inaction does harm to both society and science. In contrast, when we take steps to improve research practices in our roles as authors, reviewers, and editors, we can align our methods with the goals that inspired our work in the first place. 


\section{References}

Adams, G. (2005) The cultural grounding of personal relationship: Enemyship in North American and West African worlds. Journal of Personality and Social Psychology, 88, 948968. https://doi.org/10.1073/pnas.1817706116

Anderson, S. F., Kelley, K., \& Maxwell, S. E. (2017). Sample-size planning for more accurate statistical power: A method adjusting sample effect sizes for publication bias and uncertainty. Psychological Science, 28(11), 1547-1562.

APS Member Demographics. (2018). Psychological Science.

https://www.psychologicalscience.org/pdf/2018_APS_Member_Demographics_for_Web.pdf Ayoub, P., \& Deondra, R. (2016). Inside Higher Ed. https://www.insidehighered.com/views/2016/04/14/scholarly-importance-studying-issuesrelated-ones-own-identity-essay.

Bailey, A. H., LaFrance, M., \& Dovidio, J. F. (2020). Implicit androcentrism: Men are human, women are gendered. Journal of Experimental Social Psychology, 89, 103980. https://doi.org/10.1016/j.jesp.2020.103980

Bellamy, P. L., Mosely, D. V., Green, C. E., Neville, H., Hargons, C., Lewis, J. A., Burdine, K., Whittaker, V. A., French, B. H., Steverson, D., Adames, H. Y., Busey, C., Adam, S., Mejia, J., Ross, G., Lewis, A., Lee, L., Emerson, M., Abiodun, S., Malone, N., Chavez-Duenas, N. Y., Nadal, K., Abreu, R. L., Singh, A., Chen, G., Ratliff, K. A., Prieto, C., Saldana, M., Crowley, M., Colson, A., Trochez, J., Colocado, A., Pestana, Z., Murphy, T. H., Moskal, M., Gonzalez, K. A., Wheeler, W., Scott, L. S., Bayne, H., Gravina, N., \& Samanez-Larkin, G. (2020, June). Academics for Black Survival and Wellness. Online antiracism training course. 
Bergsieker, H. B., Shelton, J. N., \& Richeson, J. A. (2010). To be liked versus respected:

Divergent goals in interracial interactions. Journal of Personality and Social Psychology, 99(2), 248-264. https://doi.org/10.1037/a0018474

Berry, J. W. (1999). Emics and etics: A symbiotic conception. Culture \& Psychology, 5 (2), 165171. https://doi.org/10.1177/1354067X9952004

Bowleg, L. (2021). “The Master's Tools Will Never Dismantle the Master's House”: Ten Critical Lessons for Black and Other Health Equity Researchers of Color. Health Education \& Behavior, 48(3), 237-249.

Brewer, M. B., \& Crano, W. D. (2014). Research design and issues of validity. In H. T. Reis \& C. M. Judd (Eds.), Handbook of Research Methods in Social and Personality Psychology (p. 11-26). Cambridge University Press.

Brewer, W. F., \& Nakamura, G. V. (1984). The nature and functions of schemas. Center for the Study of Reading Technical Report; no. 325.

Bulhan, H. A. (2015). Stages of colonialism in Africa: From occupation of land to occupation of being. Journal of Social and Political Psychology, 3(1), 239-256.

Chaiken, S., \& Ledgerwood, A. (2012). A theory of heuristic and systematic information processing. In P. A. M. Van Lange, A. W. Kruglanski, \& E. T. Higgins (Eds.), Handbook of theories of social psychology (p. 246-266). Sage Publications Ltd. https://doi.org/10.4135/9781446249215.n13

Cheon, B. K., Melani, I., \& Hong, Y. Y. (2020). How USA-centric is psychology? An archival study of implicit assumptions of generalizability of findings to human nature based on origins of study samples. Social Psychological and Personality Science, 11(7), 928-937. https://doi.org/10.1177/1948550620927269 
Cheryan, S., \& Markus, H. R. (2020). Masculine defaults: Identifying and mitigating hidden cultural biases. Psychological Review, 127(6), 1022.https://doi.org/10.1037/rev0000209

Child Development Submissions Guidelines. (2020). Retrieved February 05, 2021, from https://www.srcd.org/research/journals/child-development/child-development-submissionguidelines

Crandall, C. S., \& Sherman, J. W. (2016). On the scientific superiority of conceptual replications for scientific progress. Journal of Experimental Social Psychology, 66, 93-99. https://doi.org/10.1016/j.jesp.2015.10.002

Crocker, J., Voelkl, K., Testa, M., \& Major, B. (1991). Social stigma: The affective consequences of attributional ambiguity. Journal of Personality and Social Psychology, 60(2), 218-228. https://doi.org/10.1037/0022-3514.60.2.218

Danbold, F., \& Unzueta, M. M. (2020). Drawing the diversity line: Numerical thresholds of diversity vary by group status. Journal of Personality and Social Psychology, 118(2), 283306. https://doi.org/10.1037/pspi0000182

da Silva Frost, A., \& Ledgerwood, A. (2020). Calibrate Your Confidence in Research Findings: A Tutorial on Improving Research Methods and Practices. https://doi.org/10.31234/osf.io/6uxkb

de Vos, A. (2020). The problem of 'colonial science'. Retrieved February 05, 2021, from https://www.scientificamerican.com/article/the-problem-of-colonial-science/

Dawson, E., Gilovich, T., \& Regan, D. T. (2002). Motivated Reasoning and Performance on the was on Selection Task. Personality and Social Psychology Bulletin, 28(10), 1379-1387. https://doi.org/10.1177/014616702236869 
DeJesus, J. M., Callanan, M. A., Solis, G., \& Gelman, S. A. (2019). Generic language in scientific communication. Proceedings of the National Academy of Sciences, 116(37), 18370-18377.

Devos, T., \& Banaji, M. R. (2005). American= white?. Journal of Personality and Social Psychology, 88(3), 447. DOI: 10.1037/0022-3514.88.3.447

Dovidio, J. F., Gaertner, S. L., \& Pearson, A. R. (2017). Aversive racism and contemporary bias. In C. G. Sibley \& F. K. Barlow (Eds.), The Cambridge Handbook of the Psychology of Prejudice (p. 267-294). Cambridge University Press. https://doi.org/10.1017/9781316161579.012

Dupree, C. H., \& Kraus, M. W. (2020). Psychological science is not race neutral. Perspectives on Psychological Science, 1745691620979820.

Faul, F., Erdfelder, E., Buchner, A. (2005). Statistical power analyses using G*Power 3.1: Tests for correlation and regression analyses. Behavior Research Methods 41, 1149-1160 . https://doi.org/10.3758/BRM.41.4.1149

Flaskerud, J. H. (2012). Cultural Bias and Likert-Type Scales Revisited. Issues in Mental Health Nursing, 33(2), 130-132. https://doi.org/10.3109/01612840.2011.600510

Garay, M. M., \& Remedios, J. (2021). A conceptual review of Whiteness and multiraciality in social psychology. Social and Personality Psychology Compass, e12642.

Gelman A., \& Carlin J.(2014). Beyond Power Calculations: Assessing Type S (Sign) and Type M (Magnitude) Errors, Perspectives on Psychological Science. 9(6), 641-651. https://doi.org/10.1177/1745691614551642

Gelman A., \& Loken E. (2014). The Statistical Crisis in Science, American Scientist. https://www.americanscientist.org/article/the-statistical-crisis-in-science 
González-Márquez, M., \& Tennant, J. (2019). Pragmatic steps for inclusivity in modern scholarly research. Open Science MOOC. Retrieved from https://opensciencemooc.eu/community/2019/10/17/inclusivity/

Griffin D., \& Tversky A. (1992). The weighing of evidence and the determinants of confidence, Cognitive Psychology. 24(3). 411-435. https://doi.org/10.1016/0010-0285(92)90013-R

Guthrie, R. V. (2004). Even the rat was white: A historical view of psychology. Pearson Education.

Helms, J. E., Jernigan, M., \& Mascher, J. (2005). The meaning of race in psychology and how to change it: A methodological perspective. American Psychologist, 60(1), 27. https://doi.org/10.1037/0003-066X.60.1.27

Henrich, J., Heine, S., \& Norenzayan, A. (2010). The weirdest people in the world? Behavioral and Brain Sciences, 33(2-3), 61-83. doi:10.1017/S0140525X0999152X

Higgins, T. E., \& Rholes W. S. (1976). Impression formation and role fulfillment: A "holistic reference" approach. Journal of Experimental Social Psychology, 12, 422-435. https://doi.org/10.1016/0022-1031(76)90075-5

Hirt, E. R., Mcdonald, H. E., \& Erickson, G. A. (1995). How Do I Remember Thee? The Role of Encoding Set and Delay in Reconstructive Memory Processes. Journal of Experimental Social Psychology, 31(5), 379-409. https://doi.org/10.1006/jesp.1995.1017

Hoenig M. J., \& Heisey M. D., (2001). The Abuse of Power: The Pervasive Fallacy of Power Calculations for Data Analysis, The American Statistician, 55(1), 19-24. https://doi.org/10.1198/000313001300339897 
Hruschka, D. J., Munira, S., Jesmin, K., Hackman, J., \& Tiokhin, L. (2018). Learning from failures of protocol in cross-cultural research. Proceedings of the National Academy of Sciences, 115(45), 11428-11434.https://doi.org/10.1073/pnas.1721166115

Jones, B. C., DeBruine, L. M., Flake, J. K., Liuzza, M. T., Antfolk, J., Arinze, N. C., ... \& Sirota, M. (2021). To which world regions does the valence-dominance model of social perception apply?. Nature Human Behaviour, 1-9.

Judd, C. M., Westfall, J., \& Kenny, D. A. (2012). Treating stimuli as a random factor in social psychology: A new and comprehensive solution to a pervasive but largely ignored problem. Journal of Personality and Social Psychology, 103, 54.

Judd, C. M., Westfall, J., \& Kenny, D. A. (2017). Experiments with more than one random factor: Designs, analytic models, and statistical power. Annual Review of Psychology, 68, 601-625.

Kendi, I. X. (2017). Stamped from the beginning: The definitive history of racist ideas in America. Random House.

Klayman, J., \& Ha, Y.-w. (1987). Confirmation, disconfirmation, and information in hypothesis testing. Psychological Review, 94(2), 211-228. https://doi.org/10.1037/0033-295X.94.2.211

Klein, R. A., Vianello, M., Hasselman, F., Adams, B. G., Adams Jr, R. B., Alper, S., ... \& Sowden, W. (2018). Many Labs 2: Investigating variation in replicability across samples and settings. Advances in Methods and Practices in Psychological Science, 1(4), 443-490.

Kuntsman, J.W., Tuscherer, T., Trawalter, S., \& Lloyd, E. P. (2016). What Lies Beneath? Minority Group Members' Suspicion of White s’ Egalitarian Motivation Predicts Responses to Whites' Smiles. Society for Personality and Social Psychology, 42, 1193-1205. https://doi.org/10.1177/0146167216652860 
Lakens D. (2017). Equivalence Tests: A Practical Primer for t Tests, Correlations, and MetaAnalyses, Social Psychological and Personality Science, 8, 355-362. https://doi.org/10.1177/1948550617697177

Lane P. S., \& Hennes P. E., (2017). Power struggles: Estimating sample size for multilevel relationships research, Journal of Social and Personal Relationships. 35, 7-31. https://doi.org/10.1177/0265407517710342

Ledgerwood, A. (2018). The preregistration revolution needs to distinguish between predictions and analyses. Proceedings of the National Academy of Sciences.

Ledgerwood, A. (2019). New developments in research methods. In E. J. Finkel \& R. F. Baumeister (Eds.), Advanced Social Psychology (pp. 39-61). Oxford University Press.

Ledgerwood, A., Pickett, C., Navarro, D., Remedios, J. D., \& Lewis, N. A., Jr. (in press). The Unbearable Limitations of Solo Science: Team Science as a Path for more Rigorous and Relevant Research. Behavioral and Brain Sciences.

Ledgerwood, A., Hudson, S. T. J., Lewis, N.A., Jr., Maddox, K. B., Pickett, C. L., Remedios, J. D., Cheryan, S., Diekman, A. B., Dutra, N. B., Goh, J. X., Goodwin, S. A., Munakata, Y., Navarro, D. J., Onyeador, I. N., Srivastava, S., \& Wilkins, C. L. (2021). The pandemic as a portal: Reimagining psychological science as truly open and inclusive. Manuscript submitted for publication. DOI: 10.31234/osf.io/gdzue

Leong, F. T. L., Leung, K., \& Cheung, F. M. (2010). Integrating cross-cultural psychology research methods into ethnic minority psychology. Cultural Diversity and Ethnic Minority Psychology, 16 (4), 590-597. https://doi.org/10.1037/a0020127 
Leventhal, H., Patrick-Muller, L., \& Leventhal, E. A. (1998). It's long-term stressors that take a toll: Comment on Cohen et al. (1998). Health Psychology, 17(3), 211213. https://doi.org/10.1037/0278-6133.17.3.211

Lewis, N. A., Jr., \& Sekaquaptewa, D. (2016). Beyond Test Performance: A Broader View of Stereotype Threat. Current Opinion in Psychology, 11, 40-43.

Likert, R. (1932). A technique for the measurement of attitudes. Archives of Psychology, 22 140, 55. https://psycnet.apa.org/record/1933-01885-001

Ma, D. S., Correll, J., \& Wittenbrink, B. (2015). The Chicago face database: A free stimulus set of faces and norming data. Behavior Research Methods, 47(4), 1122-1135. https://doi.org/10.3758/s13428-014-0532-5

Ma, D. S., Kantner, J., \& Wittenbrink, B. (2020). Chicago Face Database: Multiracial expansion. Behavior Research Methods, 1-12. https://doi.org/10.3758/s13428-020-01482-5

Majid, A., Bowerman, M., Kita, S., Haun, D. B., \& Levinson, S. C. (2004). Can language restructure cognition? The case for space. Trends in Cognitive Sciences, 8(3), 108-114. https://doi.org/10.1016/j.tics.2004.01.003

Matsumoto, D. \& van de Vijver, F. J. R. (Eds.) (2010). Cross-cultural research methods in psychology. Cambridge University Press. https://doi.org/10.1017/CBO9780511779381

McCormick-Huhn, K., Warner, L. R., Settles, I. H., \& Shields, S. A. (2019). What if psychology took intersectionality seriously? Changing how psychologists think about participants. Psychology of Women Quarterly, 43, 445-456.

Medin, D., Bennis, W., \& Chandler, M. (2010). Culture and Home-field Disadvantage. Perspectives on Psychological Science, 5(6), 708-713. https://doi.org/10.1177\%2F1745691610388772 
Mendoza A., Gollwitzer M. P., \& Amodio M. D., (2010). Reducing the Expression of Implicit Stereotypes: Reflexive Control Through Implementation Intentions. Personality and Social Psychology Bulletin, 36, 512-523. https://doi.org/10.1177/0146167210362789

Moshontz, H., Campbell, L., Ebersole, C. R., IJzerman, H., Urry, H. L., Forscher, P. S., ... Chartier, C. R. (2018). The Psychological Science Accelerator: Advancing Psychology through a Distributed Collaborative Network. Advances in Methods and Practices in Psychological Science, 1(4) , 501-515. https://doi.org/10.1177/2515245918797607

Morris, M. W., Leung, K., Ames, D. \& Lickel, B. (1999). Views from inside and outside: Integrating emic and etic insights about culture and justice judgment. Academy of Management Review, 24 (4), 781-796. https://doi.org/10.2307/259354

Nelson, J.C., Adams, G., \& Selter P. S. (2021). The Marley Hypothesis: Denial of Racism Reflects Ignorance of History. Association for Psychological Science, 24(2), 213-2018. https://doi.org/10.1177\%2F0956797612451466

Oosterhof, N. N., \& Todorov, A. (2008). The functional basis of face evaluation. Proceedings of the National Academy of Sciences, 105(32), 11087-11092. https://doi.org/10.1073/pnas.0805664105

Pauker, K., Apfelbaum, E. P., \& Spitzer, B. (2015). When Societal Norms and Social Identity Collide: The Race Talk Dilemma for Racial Minority Children. Social Psychological and Personality Science, 6, 887-895. https://doi.org/10.1177\%2F1948550615598379

Perry, S.P., Skinner, A.L. \& Abaied, J.L. (2019), Bias Awareness Predicts Color Conscious Racial Socialization Methods among White Parents. Journal of Social Issues, 75, 1035-1056. https://doi.org/10.1111/josi.12348 
Purdie-Vaughns, V., \& Eibach, R. P. (2008). Intersectional invisibility: The distinctive advantages and disadvantages of multiple subordinate-group identities. Sex Roles, 59(5-6), 377-391. DOI 10.1007/s11199-008-9424-4

Puckett, J. A., Brown, N. C., Dunn, T., Mustanski, B., \& Newcomb, M. E. (2020). Perspectives from transgender and gender diverse people on how to ask about gender. LGBT Health, 7(6), 305-311. https://doi.org/10.1089/lgbt.2019.0295

Remedios, J.D., \& Snyder, S.H. (2015). Where Do We Go From Here? Toward an Inclusive and Intersectional Literature of Multiple Stigmatization. Sex Roles 73, 408-413. https://doi.org/10.1007/s11199-015-0543-4

Rivera, G. N., Salter, P. S., Friedman, M., Crist, J., \& Schlegel, R. J. (2021). When race trumps political ideology: Black teachers who advocate for social responsibility are penalized by both liberals and conservatives. Personality and Social Psychology Bulletin, 0146167221994025.

Roberts, S. O., Bareket-Shavit, C., Dollins, F. A., Goldie, P. D., \& Mortenson, E. (2020). Racial inequality in psychological research: Trends of the past and recommendations for the future. Perspectives on Psychological Science, 15(6), 1295-1309. https://doi.org/10.1177/1745691620927709

Roberts, S. O., \& Rizzo, M. T. (2020). The psychology of American racism. American Psychologist. Advance online publication. https://doi.org/10.1037/amp0000642

Sawyer, P.J., Major, B., Casad, B.J., Townsend, S. S. M., \& Mendes, W. B. (2012). Discrimination and the Stress Response: Psychological and Physiological Consequences of Anticipating Prejudice in Interethnic Interactions. American Journal of Public Health, 102(5), 1020-1026. https://ajph.aphapublications.org/doi/abs/10.2105/AJPH.2011.300620 
Salter, P., \& Adams, G. (2013). Toward a critical race psychology. Social and Personality Psychology Compass, 7(11), 781-793. https://doi.org/10.1111/spc3.12068

Salter, P. S., Adams, G., \& Perez M. J. (2017). Racism in the Structure of Everyday Worlds: A Cultural-Psychological Perspective. Association for Psychological Science, 27(3), 150-155. https://doi.org/10.1177\%2F0963721417724239

Sanchez, A., Coleman, C., \& Ledgerwood, A. (2021). Does temporal distance influence abstraction? A large pre-registered experiment. Social Cognition, 39, 352-365.

Sanchez C.,\& Dunning D. (2018). Overconfidence Among Beginners: Is a Little Learning a Dangerous Thing? Journal of Personality and Social Psychology, 114(1), 10-28 http://dx.doi.org/10.1037/pspa0000102

Schönbrodt, F. D., \& Perugini, M. (2013). At what sample size do correlations stabilize? Journal of Research in Personality, 47(5), 609-612. https://doi.org/10.1016/j.jrp.2013.05.009

Segall, M. H., Campbell, D. T., \& Herskovits, M. J. (1966). The influence of culture on visual perception (pp. 174-84). Indianapolis: Bobbs-Merrill.

Simons, D. J., Shoda, Y., \& Lindsay, D. S. (2017). Constraints on generality (COG): A proposed addition to all empirical papers. Perspectives on Psychological Science, 12(6), 1123-1128. https://doi.org/10.1177/1745691617708630

Skov, R.B., \& Sherman, S. J. (1986). Information-gathering processes: Diagnosticity, hypothesis-confirmatory strategies, and perceived hypothesis confirmation. Journal of Experimental Social Psychology, 22(2), 93-121. https://doi.org/10.1016/0022$\underline{1031(86) 90031-4}$

Steele, C. M. (1999, August). Thin Ice: "Stereotype Threat" and Black College Students. The Atlantic Monthly, 284(2), 44. 
Steele, C. M., \& Aronson, J. (1995). Stereotype threat and the intellectual test performance of African Americans. Journal of Personality and Social Psychology, 69(5), 797-811. https://doi.org/10.1037/0022-3514.69.5.797

Stevens, K. R., Masters, K. S., Imoukhuede, P. I., Haynes, K. A., Setton, L. A., CosgriffHernandez, E., ... \& Eniola-Adefeso, O. (2021). Fund black scientists. Cell, 184(3), 561-565. Society for Personality and Social Psychology. (2018). Out of the Lab with Brenda Major [Video]. YouTube. https://www.youtube.com/watch?v=6FRr64mm1DE

Song, H., Lewis Jr, N. A., Ballew, M. T., Bravo, M., Davydova, J., Gao, H. O., ... \& Schuldt, J. P. (2020). What counts as an "environmental" issue? Differences in issue conceptualization by race, ethnicity, and socioeconomic status. Journal of Environmental Psychology, 68, 101404. https://doi.org/10.1016/j.jenvp.2020.101404

Thalmayer, A. G., Saucier, G., Ole-Kotikash, L., \& Payne, D. (2020). Personality structure in east and west Africa: Lexical studies of personality in Maa and Supyire-Senufo. Journal of Personality and Social Psychology, 119(5), 1132. https://doi.org/10.1037/pspp0000264

Thalmayer, A. G., Toscanelli, C., \& Arnett, J. J. (2020). The neglected 95\% revisited: Is American psychology becoming less American?. American Psychologist. https://doi.org/10.1037/amp0000622

Tropp, L. R., \& Wright, S. C. (2003). Evaluations and perceptions of self, ingroup, and outgroup: Comparisons between Mexican-American and European-American children. Self and Identity, 2(3), 203-221. https://doi.org/10.1080/15298860309028

Vandello, J. A., \& Cohen, D. (1999). Patterns of individualism and collectivism across the United States. Journal of Personality and Social Psychology, 77(2), 279. 
Vinluan, A. C., \& Remedios, J. D. (2020). Who Do Multiracials Consider Part of Their Racial In-Group?. Social Psychological and Personality Science, 11(4), 522-532. https://doi.org/10.1177/1948550619876639

Wang, Y. A., \& Rhemtulla, M. (2020). Power analysis for parameter estimation in structural equation modeling: A discussion and tutorial. https://doi.org/10.31234/osf.io/pj67b

Westfall, J., Kenny, D. A., \& Judd, C. M. (2014). Statistical power and optimal design in experiments in which samples of participants respond to samples of stimuli. Journal of Experimental Psychology: General, 143(5), 2020.

Winking, J., Eastwick, P. W., Smith, L. K., \& Koster, J. (2018). Applicability of the investment model scale in a natural fertility population. Personal Relationships, 25, 497-516.

Xie, S.Y., Flake, J.K., Stolier, Freeman, \& Hehman, E. (in press). Facial impressions are predicted by the structure of group stereotypes. Psychological Science.

Yogeeswaran, K., \& Dasgupta, N. (2010). Will the "real" American please stand up? The effect of implicit national prototypes on discriminatory behavior and judgments. Personality and Social Psychology Bulletin, 36(10), 1332-1345.https://doi.org/10.1177/0146167210380928 